\title{
MYOSTATIN LEVEL IN CRF PATIENTS WITH AND WITHOUT POST-HCV CIRRHOSIS AND ITS CORRELATION WITH BMI
}

\author{
By \\ MOHAMED MAGDY SALAMA ${ }^{1}$, MOHAMED ALI MAKHLOUF ${ }^{1}$, \\ MOHAMED MOHEI EL BADRY ${ }^{2^{*}}$, HEBA MAHMOUD ELFOULY ${ }^{3}$, \\ And HAGAR AHMED ELESSAWY ${ }^{1}$
}

Department of Internal Medicine, Faculty of Medicine, Ain Shams University, Cairo, Department of Tropical Medicine and Gastroenterology, Faculty of Medicine, Aswan

University, Aswan, and Department of Internal Medicine, Hammoul Central Hospital, Kafr El Shaikh, Egypt ('Correspondence: melbadry2002@yahoo.com)

\section{Abstract}

Chronic kidney disease (CKD) is a progressive condition that may negatively affect musculoskeletal health. Secondary sarcopenia due to CKD may be associated with mobility limitations and elevated fall risk. Thus, it is important to investigate surrogate methods that enable the assessment of muscle mass. Myostatin regulates synthesis and degradation of skeletal muscle proteins and is associated with the development of sarcopenia. This study assessed the myostatin level in CKD patients and correlated with body mass index. This a prospective case control study, carried out on 30 Egyptian patients with CKD (15 have post-HCV cirrhosis) attended for dialysis in Al-Hammoul Central Hospital and 15 healthy controls. All patients were subjected to detailed medical history and through clinical examination, abdominal ultrasound and determination of serum myostatin level. The mean, standard deviation and chi-square were calculated by SPSS. ROC curves were conducted to test the discriminative value of myostatin level on sarcopenia and to detect the cut-off points. The results showed significant increase in the serum myostatin level in hemodialysis patients. Myostatin level in post-HCV cirrhotic patients was insignificantly higher than in non-cirrhotic patients. There was a significant negative correlation between serum myostatin level and BMI and skeletal muscle index (SMI). ROC curve showed that a cut off value of $39.05 \mathrm{ng} / \mathrm{ml}$ can detect sarcopenia in hemodialysis patients.

Key-words: Chronic renal disease, Myostatin, HCV, BMI, Cirrhosis.

\section{Introduction}

Chronic kidney disease (CKD) is a progressive condition that might negatively affect musculoskeletal health. Secondary sarcopenia due to CKD may be accompanied with elevated fall risk and mobility limitations (Hernand et al, 2018). The loss of muscle mass, in addition to the impact of poor body composition on muscle strength and mobility status are necessary for classification and staging of sarcopenia (Cruz-Jentoft, et al, 2010). Patients with CKD are prone to muscle wasting. Thus, it is important to investigate suitable methods for the clinical assessment of muscle mass (Giglio et al, 2018). The hemodialysis was found to stimulate protein degradation and reduced protein synthesis for $2 \mathrm{hr}$ following dialysis, suggested that a process causing protein loss was initiated by this therapy and persisted. Increasing the intake of protein and calories could improve protein turnover but, it did not fully correct the responses to hemodialysis (Cruz-Jentoft et al, 2010). Diagnosis of sarcopenia could be achieved by measuring mid-arm circumference which is a simple anthropometric method that reflected the muscle mass amount by deducting amount of measured fat in the triceps and bone width. Sarcopenia can be diagnosed when this value is below the 10th percentile from a reference population (Duarte-Roio et al, 2015). In the same context, Up to $70 \%$ of patients having an advanced liver disease might develop sarcopenia. Liver cirrhosis is associated with an altered glucose metabolism, ketogenesis, lipid oxidation and protein catabolism, leading to the loss of muscle and adipose tissue. The gastrointestinal dysfunction of cirrhotic patients results in insufficient nutrients intake and is responsible for muscle weakness thus limiting physical exercise and perpetuating reduction of muscle mass (Panziani and Gasbarrini, 2018). 
Myostatin is a member of "transforming growth factor $\beta$ family", which regulates skeletal muscle proteins' synthesis and degradation, and it is associated with sarcopenia. It also regulates the proliferation and differentiation of myoblasts (Langley et al, 2002). It acts in a paracrine fashion to maintain satellite cells within muscle in a quiescent state. Insulin-like growth factor 1 (IGF1) has dual action such that it inhibits myostatin as well as it stimulates mammalian target of rapamycin (mTOR), through stimulating muscle growth by both activating satellite cells and muscle protein synthesis (Thapalive et al, 2014). Besides, it controls the activation and proliferation of the stem cells of skeletal muscle. Myostatin is upregulated in the skeletal muscle of CKD patients and is thought to be involved in the development of uremic sarcopenia. But, serum myostatin levels were rarely determined and the relationship between myostatin levels with clinical and metabolic factors remain unknown (Yamada, 2016). Complex biology of myostatin and its inhibition as an effective way to counter sarcopenia, and the challenges facing its clinical translation were of great interest by authors in the last decade.

This study aimed to assess the myostatin level in chronic renal failure patients with or without cirrhosis, and to study its correlation with body mass index in those patients as compared to matched controls.

\section{Subjects and Methods}

Patient recruitment: It is a prospective case control study was carried out on 30 Egyptian patients with chronic kidney disease (15 patients had post-HCV cirrhosis) who attended for dialysis in $\mathrm{Al}$ Hammoul Central Hospital and 15 Egyptian apparently healthy subjects as a control group. It was conducted during the period from June 2018 to May 2019. The age of the included subjects ranged from 25 -50 years. They were divided into 3 groups; GI: 15 hemodialysis patients without cirrhosis or HCV, GII: 15 hemodialysis patients with post-HCV cirrhosis and GIII: 15 healthy controls with cross matched age and sex.
Patients with cirrhosis (Child B or C), chronic pulmonary disease, autoimmune disease or cancer were excluded. The study was done according to the ethical guidelines of the 1975 Declaration of Helsinki and approval by Institutional Review Board (IRB) for human subject research at Ain Shams University. A written informed consent was obtained from all enrolled participants before enrolment to the study.

Study design: It is a prospective observantional case control study of patients with chronic renal failure on hemodialysis, Al Hammoul Central Hospital, Kafr El-Shaikh Governorate. All participants were subjected to complete history taking with special emphasis on causes of the chronic kidney disease (hypertension and diabetes mellitus nephrosclerosis, renal vascular disease, and chronic glomerulonephritis...etc.) history of jaundice, disturbed conscious level, bleeding tendency, hematemesis or melena, lower limbs edema, and weight loss. History of Comorbid illnesses and drugs were obtained by patient interventions and confirmed from medical records later. Physical examination was done and all were evaluated for their height, weight, body mass index (BMI). Weight was measured immediately after hemodialysis (HD) session. BMI was calculated as weight divided by height squared $(\mathrm{kg} / \mathrm{m} 2)$. Measurement of skeletal muscle index (SMI); mid-arm circumference was reflected amount of muscle mass by deducted the amount of measured fat in triceps and bone width. Diagnosis of sarcopenia was considered if the value was below $10^{\text {th }}$ percentile from a reference standard (Duarte-Roio et al, 2018). Routine lab examinations were done as CBC (Schiff et al, 2011), kidney function test; blood urea, serum creatinine (Delanphe and Speekaert, 2011), liver function test; ALT, AST, Albumin, PT, INR (Dufour et al, 2011), $\mathrm{Na}, \mathrm{K}, \mathrm{Ca}, \mathrm{Po}_{4}$ electrolytes (Weissman and Pileggi, 1974) and viral markers serological tests for HCVAb by ELISA (Villar et al, 2014). To determine serum myostatin level, blood samples were collected before midweek HD 
session and plasma was separated within 30 min by centrifuging at $3600 \mathrm{r} / \mathrm{min}$ for $15 \mathrm{~min}$ at room temperature and frozed at $-80^{\circ} \mathrm{C}$ until myostatin analysis. Serum myostatin levels were determined by commercial ELISA kit (Sun Red, Biotechnology Co, Shanghai, 201-12-0404). Kit used a double-antibody sandwich ELISA. Chroma of color and concentration of human substance myostatin of sample correlated positively (Tab. 1).

Table 1: Standard dilution

\begin{tabular}{|l|l|l|}
\hline $1200 \mathrm{ng} / \mathrm{L}$ & StandardNo.5 & $120 \mu \mathrm{l}$ Original Standard $+120 \mu \mathrm{l}$ Standard diluents \\
\hline $600 \mathrm{ng} / \mathrm{L}$ & Standard No.4 & $120 \mu \mathrm{l}$ Standard No.5 $+120 \mu 1$ Standard diluents \\
\hline $300 \mathrm{ng} / \mathrm{L}$ & Standard No.3 & $120 \mu \mathrm{l}$ Standard No.4 $+120 \mu 1$ Standard diluents \\
\hline $150 \mathrm{ng} / \mathrm{L}$ & Standard No.2 & $120 \mu \mathrm{l}$ Standard No.3 $+120 \mu 1$ Standard diluents \\
\hline $75 \mathrm{ng} / \mathrm{L}$ & Standard No.1 & $120 \mu \mathrm{l}$ Standard No.2 $+120 \mu 1$ Standard diluents \\
\hline
\end{tabular}

First, $50 \mu$ of standards and $50 \mu l$ of Streptavidin-HRP were added to each well. $40 \mu \mathrm{l}$ of samples, then $10 \mu \mathrm{l}$ MSTN antibody and $50 \mu \mathrm{l}$ of Streptavidin-HRP were added to each sample well. Sealing membrane was applied, gently shaking was done and plate was incubated for 60 minutes at $37^{\circ} \mathrm{C}$. Wash buffer $(30 \times)$ was diluted 30 times with 380 $\mathrm{ml}$ distilled water. Membrane was carefully removed, and liquid was drained. Chromogen solution A $50 \mu \mathrm{l}$, then chromogen solution B $50 \mu$ were added to each well, incubated for $10 \mathrm{~min}$ at $37^{\circ} \mathrm{C}$ in dark. Stop solution $50 \mu$ was added into each well to stop the reaction (blue change into to yellow color immediately). The optical density (OD) was measured under $450 \mathrm{~nm}$ wavelength within 15 min after adding the stop solution.

Abdominal ultrasonography was done using Siemens Sonolone Sienna sonography for all to detect kidney size, shape and liver cirrhosis, Splenomegaly, evidence of portal hypertension or ascites. Child score was determined according to Modified Child-Pugh classification of liver disease severity according to the ascites degree, plasma concentrations of bilirubin and albumin, prothrombin time, and the encephalopathy degree [5-6 = grade A, 7-9 = grade B, 10-15 =grade C] (Durand and Valla, 2005).

\section{Results}

The demographic characteristics showed the mean age of non-cirrhotic (GI) was 38.3 years; 6 patients of them were male and the mean age of post-HCV (GII) was 37.7 years; 7 patients of them were male however the mean age of the control (GIII) was 35.7 years; 5 individuals of them were male.
In the present study, the commonest cause of chronic renal failure in GI \& GII was diabetic nephropathy (20\%), but hypertensive nephropathy was $13.3 \%$, glomerulonephritis was $11.1 \%$ and hereditary causes were $6.7 \%$. The interstitial nephritis and unknown causes were $4.4 \%$ with same distribution among patients. Polycystic kidney, renal vascular disease and pyelonephritis were $2.2 \%$ but with different distributions among patients.

The mean \pm SD BMI in GI, GII \& GIII was $17.3 \pm 3.9,15.7 \pm 3.6 \& 22.7 \pm 2.0$, respectively. The skeletal muscle mass index in GI, GII \& GIII was $26.4 \pm 5.7,23.3 \pm 4.7$ \& $35.3 \pm 3.1$, respectively. BMI and SMI, did not show significant difference among the groups, but significant difference was between GI \& GII ( $\mathrm{P}<0.001$ by Tukey's test). Myostatin level in GI, GII \& GIII was $42.3 \pm 6.1,43.9 \pm 8.8 \& 4.0 \pm 0.9$, respectively, without significant difference between GI \& GII was found, but GIII showed significant difference with both GI \& GII $(p<0.001)$. Myostatin level showed a significant negative correlation with BMI $(\mathrm{r}=-0.646$, $\mathrm{p}<0.001)$ and SMI $(\mathrm{r}=-0.682, \mathrm{p}<0.001)$, but without significant correlation between myostatin level and age.

The ROC curves showed that myostatin level could be a valuable marker in prediction of sarcopenia in hemodialysis patients. The cut-off value was $39.05 \mathrm{ng} / \mathrm{ml}$, with $94.1 \%$ sensitivity, $85.7 \%$ specificity, $80.0 \%$ positive predictive value, $96.0 \%$ negative predictive value, and $88.9 \%$ accuracy.

\section{Discussion}

Patients with chronic kidney disease are subjected to muscle wasting. So, it was imp- 
ortant to investigate surrogate methods that enable the assessment of muscle mass loss in the clinical setting (Giglio et al, 2018). Myostatin is the major negative regulator of growth that is highly enriched in skeletal muscle (Thapalive et al, 2014). There was great interest in myostatin as a potential mediator of sarcopenia as well as a therapeutic target.

In the present study, there was no significant difference between the studied groups regarding age and gender. This ensured that neither age and sex effected on the study results nor reflected the homogeneity of the patient sample. But in this study, there was female predominance with female/male ratio of 27/18. Female patients with CKD were more as compared to male counterparts. The present data agreed with others as old-aged female was CKD risky (Zaman et al, 2016). This agreed also with Han et al. (2011) who assessed the serum myostatin levels and grip strength among patients on maintenance hemodialysis compared to controls, but did not find difference in age and sex between patients and controls.

In the present study, there was significant decrease in the mean platelet count values in CKD with post-HCV cirrhosis patients compared to controls. This agreed with Han et al. (2011). HCV infection was the etiology of cirrhosis in all present patients. McCormick and Murphy (2000) showed high levels of platelets associated IgG in cirrhotic patients with thrombocytopenia and this IgG is believed to represent binding of anti-platelets antibody and/or IgG immune complexes to the platelets, removed by the splenic and hepatic reticuloendothelial system and destroyed. This suggested that the present thrombocytopenia patients could be immune mediated more over than decreased production of platelets due to thrombocytopenia in cirrhotic patients. This agreed with McCormick and Murphy (2000).

In the present study, the etiology of chronic renal failure showed wide variations; the commonest cause was diabetic nephropathy
(20\%, 4 patients in GI \& 5 patients in GII). Hypertensive nephropathy was represented by $13.3 \%$ (3 patients in each of GI \& G II). But, glomerulonephritis was represented by $11.1 \%$ ( 3 patients in GI \& 2 patients in GII), hereditary causes were represented by $6.7 \%$ (1 patient in GI \& 2 patients in GII). However, the interstitial nephritis and unknown causes had the same value $(4.4 \%)$ and the same distribution among patients (1 patient in each of GI \&GII). Polycystic kidney, renal vascular disease and pyelonephritis had the same value $(2.2 \%)$ but with different distribution among them (1 patient in GI, 1 patient in GII \& 1 patient in GI, respectively). This agreed with Japanese Society of Nephrology (2014) who reported that the most common recognized cause of CKD was diabetes mellitus. Others included idiopathic cause often associated with small kidneys on renal ultrasound, hypertension, and/or glomerulonephritis. This caused about $75 \%$ of all adults. Historically, kidney disease was classified according to the part of the kidney anatomy involved. This disagreed with Ishikawa et al. (2018) who found that the commonest origin of CKD was sarcopenia in the chronic renal disease patients, which diuretics effect was benign nephrosclerosis (48.8\%), chronic glomerulonephritis (19.6\%) \& diabetic nephropathy (13.1\%). Koyun et al. (2018) reported the causes of chronic kidney disease were hypertensive nephrosclerosis and renal vascular (25\%), diabetic nephropathy (12\%), chronic glomerulonephritis (11\%), pyelonephritis $(7 \%)$, polycystic kidney disease $(6 \%)$, or unknown causes $(39 \%)$.

In the present study, body mass index showed a significant difference in comparison between groups with the lowest values of mean and standard deviation reported in GII (15.7 \pm 3.6$)$ with $\mathrm{P}<0.001$. The reason of low BMI in CRF patients could be that CKD patients usually develop anemia with decline in their nutritional status (Mafra et al, 2008). This agreed with Han et al. (2011) who found that patients receiving hemodialysis had lower BMI and weaker grip strength. 
Also, Zaman et al. (2017) who reported that the measure of BMI was found lower among the CKD patients' than the non-CKD group.

In the present study, there was high significant difference $(p<0.001)$ among patients regarding SMI with the lowest level in patients of post-HCV cirrhosis (23.3 \pm 4.7$)$. They submitted that low-muscle mass was secondary to the effects of low muscle strength but other factors such as contractile quality, neural activation, systemic inflammation, and underestimated nutritional disorders may play a more important part (Isoyama et al, 2014). This agreed with Du et al. (2005) who reported that skeletal muscle wasting and cachexia are common in patients receiving hemodialysis. Also, Han et al. (2011) reported that body weight, BMI, muscle mass, and grip strength had a downward tendency in HD patients, with the muscle mass higher in normal controls than that in dialysis ones.

In the present study, sarcopenia was significantly different among groups. It was significantly higher in GI but the highest one was in GII (chronic kidney disease with HCV positive patients). The sarcopenia incidence in all was $(53.3 \%, 60.0 \%, 00.0 \%$ respectively) with total 17 patients out of 45 subjects (37.8\%). Muscle turnover increases during HD, resulting in net increase in catabolism (Raj et al, 2014). Cross-sectional imaging studies reported that the prevalence of sarcopenia was 30\%-70\% among patients with cirrhosis (Kim and Jang, 2015).

In the present study, there was a highly significant difference of myostatin level between groups $(\mathrm{P}<0.001)$, with the highest one in GII (43.9 \pm 8.8$)$. This agreed with Koyun et al. (2018) who found significant serum myostatin elevated level as compared to controls $(40.1 \pm 8.3$ vs. $2.5 \pm 2.4 \mathrm{ng} / \mathrm{ml}, \mathrm{P}<$ 0.001). This agreed with Yano et al. (2015) who found that plasma myostatin level was elevated in an early CKD stage due to sarcopenia progression. But, the result disagreed with Han et al. (2011) who found lower serum myostatin levels in $60 \mathrm{HD}$ patients than healthy ones.
In the present study, there was a negative relation between myostatin level and both BMI \& SMI. This agreed with Ju and Chen (2012) and Wang et al. (2012) they found contradictory results between serum myostatin levels and skeletal muscle mass, measured by various methods, in diabetes mellitus, chronic obstructive pulmonary disease, heart failure and end-stage liver disease. This disagreed with Han at al. (2011) who found the myostatin level did not correlate with sex, height, body weight, BMI and muscle mass.

In the present study, myostatin level at a cutoff value $39.05 \mathrm{ng} / \mathrm{ml}$ in detecting sarcopenia gave a sensitivity $94.1 \%$, specificity $85.7 \%$, PPV $80.0 \%$, NPV $96.0 \%$, \& accuracy $88.9 \%$. This agreed with Koyun et al. (2018) who found that HD patients had significant elevated serum myostatin level as compared to controls $(40.1 \pm 8.3$ vs. $2.5 \pm-2.4$ $\mathrm{ng} / \mathrm{ml}, \mathrm{P}<0.001$ ). Yano et al. (2015) reported that plasma myostatin level was elevated in an early stage of CKD, which could involve the sarcopenia progression. But, Han et al. (2011) found that myostatin serum and IGF-1 level in hemodialysis patients had lower myostatin level, IGF-1, lower grip strength and lower BMI compared with controls.

\section{Conclusion}

The serum myostatin level was significantly higher in hemodialysis patients than controls, particularly in post-HCV cirrhotic than non-cirrhotic ones. Serum myostatin level was significantly related to sarcopenia pathogenesis as a predictor marker.

\section{References}

Carrero, JJ, Stenvinkel, P, Cuppari, L, et al, 2013: Etiology of the protein-energy wasting syndrome in chronic kidney disease: a consensus statement from the International Society of Renal Nutrition and Metabolism (ISRNM). J. Ren. Nutr. 23, 2:77-90.

Cruz-Jentoft, AJ, Baeyens, JP, Bauer, JM, et al, 2010: Sarcopenia: European consensus on definition and diagnosis: Report of the European Working Group on Sarcopenia in Older People. Age Ageing 39:412-23.

Delanghe, JR, Speeckaert, MM, 2011: Creatinine determination according to Jaffe- 
what does it stand for? NDT-Plus 4, 2:83-6. Du, J, Hu, Z, Mitch, WE, 2005: Molecular mechanisms activating muscle protein degradation in chronic kidney disease and other catabolic conditions. Eur. J. Clin. Invest. 35, 3:157-63.

Duarte-Rojo A, Ruiz-Margáin A, Montaño-L oza AJ, Rodríguez RU, Ferrando A, et al, 2018: Exercise and physical activity for patients with end-stage liver disease: Improving functional status and sarcopenia while on the transplant waiting list. Liver Transpl. 24, 1:122-39.

Dufour, DR, Lott, JA, Nolte, FS, Gretch, DR, Koff, RS, et al, 2000: Diagnosis and monitoring of hepatic injury. I. Performance characteristics of laboratory tests. Clin. Chem. 46:2027-49.

Durand, F, Valla, D, 2005: Assessment of the cirrhosis prognosis: Child-Pugh versus MELD. J. Hepatol. 42, 1:S100-7.

Giglio, J, Kamimura, MA, Lamarca, F, Rodrigues, J, Santin, F, et al, 2018: Association of sarcopenia with nutritional parameters, quality of life, hospitalization, and mortality rates of elderly patients on hemodialysis. J. Ren. Nutr. 28, 3:197-207.

Han, DS, Chen, YM, Lin, SY, et al, 2011: Serum myostatin levels and grip strength in normal subjects and patients on maintenance haemodialysis. Clin. Endocrinol. 75, 6:857-63.

Hernandez, H, Obamwonyi, G, Harris-Love, M, et al, 2018: Physical therapy considerations for chronic kidney disease and secondary sarcopenia. J. Funct. Morphol. Kinesiol. 3, 1: 5. doi: 10.3390/jfmk3010005. PMID: 29376141.

Isoyama, N, Qureshi, AR, Avesani, CM, et al, 2014: Comparative associations of muscle mass and muscle strength with mortality in dialysis patients. Clin. J. Am. Soc. Nephrol. 9, 10:17208.

Japanese Society of Nephrology, 2014: Clin. Exp. Nephrol. 18:346. https://doi.org/10.1007/s 10157 -014-0949-2

Ju, CR, Chen, RC. 2012: Serum myostatin levels and skeletal muscle wasting in chronic obstructive pulmonary disease. Respir. Med. 106, 1:102-8.

Kim, HY, Jang, JW, 2015: Sarcopenia in the prognosis of cirrhosis: Going beyond the MELD score. World J. Gastroenterol. 21, 25:7637-47. Koyun, Nergizoglu, G, Kir, KM, 2018: Evaluation of the relationship between muscle mass and serum myostatin levels in chronic hemodialysis patients. Saudi J. Kid. Dis. Transplant. 29, 4:809-15.
Langley, B, Thomas, M, Bishop, A, Sharma, M, et al, 2002: Myostatin inhibits myoblast differentiation by down-regulating MyoD expression. J. Biol. Chem. 277, 51:4983-40.

Mafra, D, Guebre-Egziabher, F, Fouque, D, 2008: Body mass index, muscle and fat in chronic kidney disease: questions about survival. Nephrol. Dial. Transplant. 23:2461-6.

McCormick, PA, Murphy, KM, 2000: Splenomegaly, hypersplenism and coagulation abnormalities in liver disease. Res. Clin. Gastroenterol. 14, 6:1009-31.

Ponziani, FR, Gasbarrini, A, 2018: Sarcopenia in patients with advanced liver disease. Curr. Protein Pept. Sci. 19, 7:681-91.

Raj, DSC, Zager, P, Shah, VO, et al, 2004: Protein turnover and amino acid transport kinetics in end-stage renal disease. Am. J. Physiol. Endocrinol. Metab. 286, 1:E136-43.

Schiff, ER, Maddrey, WC, Sorrell, MF, 2011: Schiff's Diseases of the Liver. Wiley-Blackwell. Thapaliya, S, Runkana, A, McMullen, MR, Nagy, LE, McDonald, C, et al, 2014: Alcoholinduced autophagy contributes to loss in skeletal muscle mass. Autophagy 10, 4:677-90

Villar, LM, Cruz, HM, Barbosa, JR, Bezerra, CS, Portilho, MM, et al, 2015: Update on hepatitis $\mathrm{B}$ and $\mathrm{C}$ virus diagnosis. Wd. J. Virol. 4, 4: 323-42.

Wang, F, Liao, Y, Li, X, et al, 2012: Increased circulating myostatin in patients with type 2 diabetes mellitus. J. Huazhong Uni. Sci. Technol. Med. Sci. 32:534-9.

Weissman, N, Pileggi, VJ, 1974: Inorganic ions. In: Henry RJ, Cannon DC, Winkelman JW (eds). Clinical chemistry: Principles and Technics. $2^{\text {nd }}$ Edn. Hagerstown: Harper and Row Yamada, S, Tsuruya, K, Yoshida, H, Tokumoto, M, Ueki, K, et al, 2016: Factors associated with the serum myostatin level in patients undergoing peritoneal dialysis: Potential effects of skeletal muscle mass and vitamin $\mathrm{D}$ receptor activator use. Calcif. Tissue Int. 99, 1:13-22.

Yano, S, Nagai, A, Isomura, M, et al, 2015: Relationship between blood myostatin levels and kidney function: Shimane Cohere Study. Plos One 10:e0141035.

Zaman, S, Hossain, N, Rahman, AE, Islam, S MS, 2016: Can glycated hemoglobin act as a reliable glycemic indicator in patients with diabetic chronic kidney disease? Evidence from the northeast of Thailand. Indonesia Med. J. 26, 2:102-8. 


\section{Explanation of figures}

Fig. 1: Pearson Correlation test between myostatin level and body mass index.

Fig. 2: Correlation between myostatin level and skeletal muscle mass index.
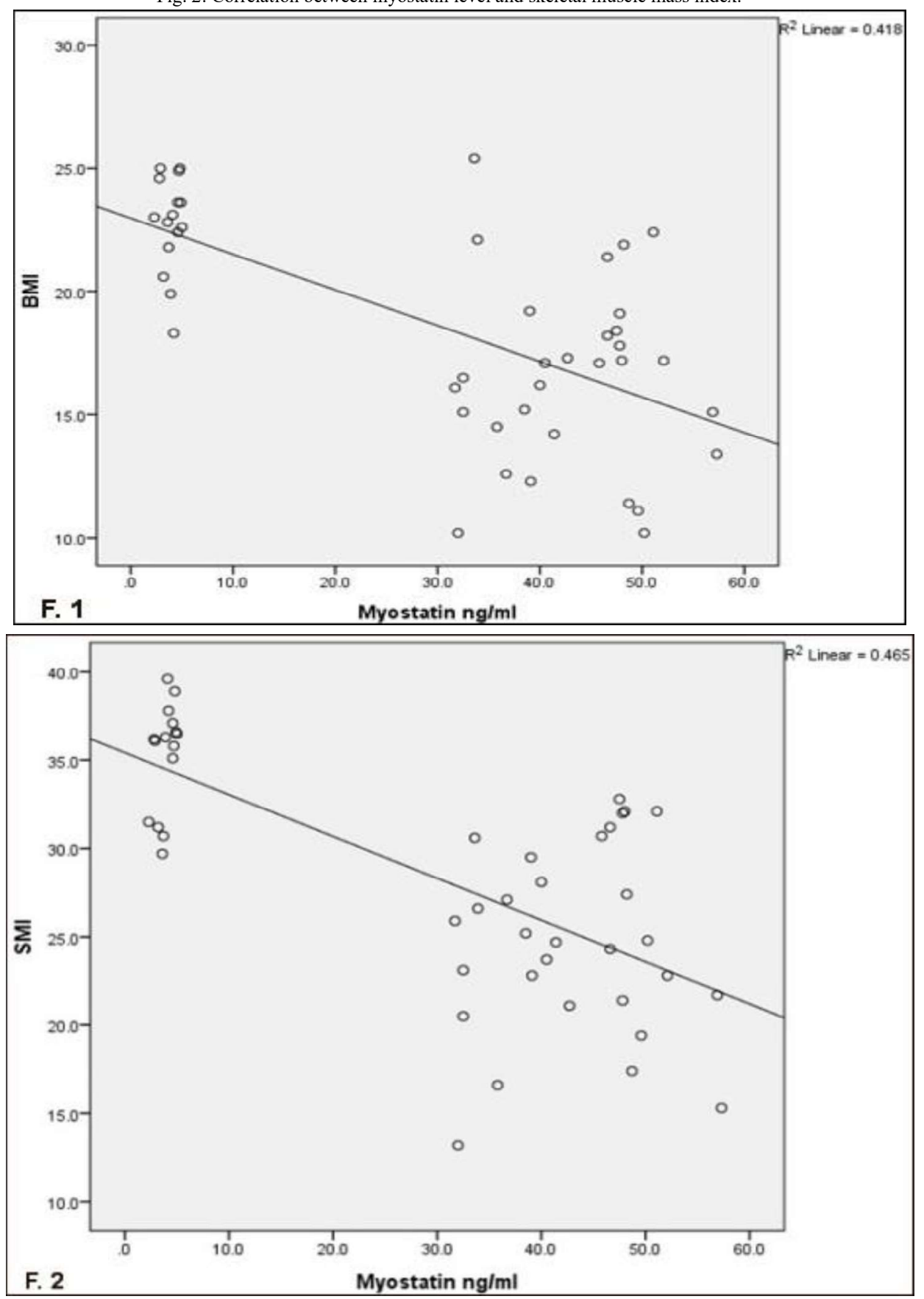